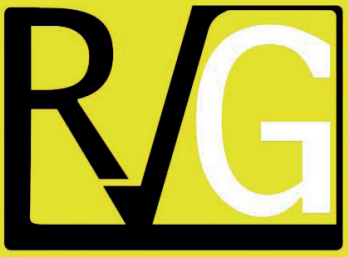

Julio - Septiembre, 2021

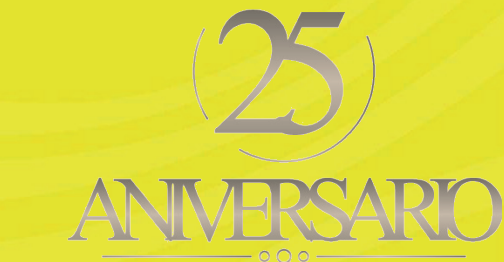

ANMERSARO
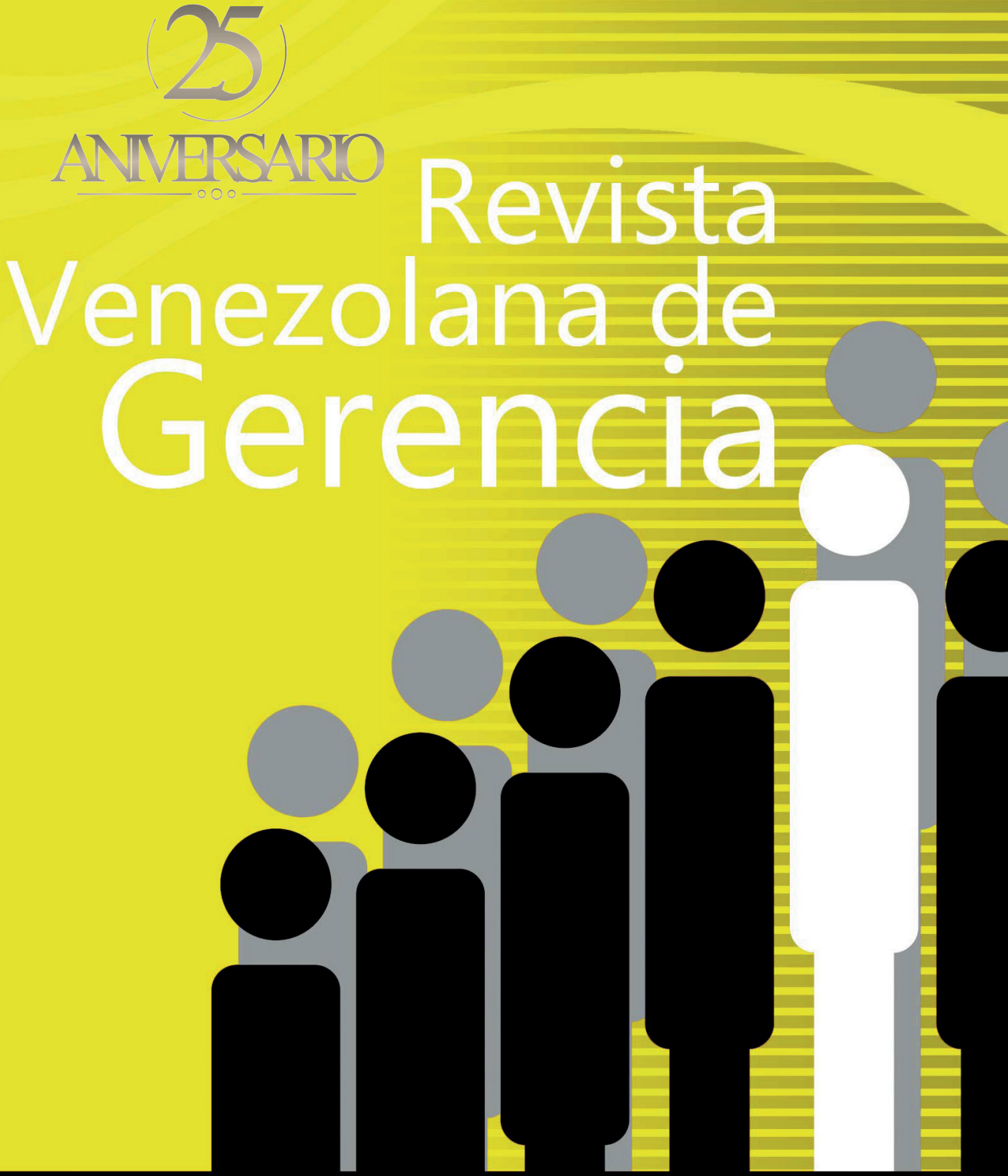

UNIVERSIDAD DEL ZULIA (LUZ)

Facultad de Ciencias Económicas y Sociales Centro de Estudios de la Empresa

ISSN 1315-99

Esta obra está bajo una licencia de Creative Comm Reconocimiento-NoComercial-Compartirlgual 3.0 Unpo http://creativecommons.org/licenses/by-nc-sa/3.0/deed.es 
COMO CITAR: Escobar Carmelo, K. R., Terry Ponte, O. F., Zavaleta Chávez, W. E., y Zárate Ruiz, G. E. (2021). Desempeño de los gobiernos subnacionales en la gestión de inversión pública del Perú. Revista Venezolana de Gerencia, 26(95), 595-609. https://doi.org/10.52080/ rvgluz.27.95.10
Universidad del Zulia (LUZ)

Revista Venezolana de Gerencia (RVG)

Año 26 No. 95 Julio-Septiembre 2021, 595-609

ISSN 1315-9984 / e-ISSN 2477-9423

\title{
Desempeño de los gobiernos subnacionales en la gestión de inversión pública del Perú
}

\author{
Escobar Carmelo, Keny Rodrigo* \\ Terry Ponte, Otto Franklin** \\ Zavaleta Chávez, Walter Enrique*** \\ Zárate Ruiz, Gustavo Ernesto****
}

\section{Resumen}

La presente investigación tuvo por finalidad analizar el desempeño de los gobiernos subnacionales en la gestión de inversión pública en Perú. El estudio fue de tipo básico, no experimental, de enfoque cualitativo; se desarrolló a través de la revisión documental de fuentes diversas pertinentes y relevantes para el objeto de investigación. Los resultados indican que los gobiernos subnacionales presentan inapropiado desempeño en la gestión de las inversiones públicas por factores diversos como capacidad de gestión, puesto que existen brechas notorias en la ejecución del gasto de inversión, personal inadecuado e intereses políticos que afectan el nivel de ejecución en años no electorales. Los gobiernos regionales presentan peor desempeño de la gestión de inversiones que los gobiernos locales ya que el decrecimiento es $5.53 \%$ y $1.30 \%$, respectivamente.

Palabras claves: Gobiernos subnacionales; inversión pública; gestión de la inversión pública; desempeño estatal.

Recibido: 23-09-20

Aceptado: 15-12-20

* Maestro en Gestión Pública, Universidad César Vallejo-Perú; Ingeniero Empresarial de profesión, especialista en inversión pública en gobiernos subnacionales. Universidad César Vallejo, Email: krescobarc19@gmail. com, Orcid: https://orcid.org/0000-0002-4849-691X

** Doctor en Gestión Pública y Gobernabilidad, Universidad César Vallejo - Perú; Magister en Administración de Negocios y Relaciones Internacionales. Contador Público y Abogado. Coordinador de la Carrera de Contabilidad. Universidad Cesar Vallejo S.A.C, Lima - Perú, Email: oterry@ucv.edu.pe, Orcid: https://orcid. org/0000-0003-0717-8023.

*** Doctor en Educación, Universidad César Vallejo - Perú; Magister en Administración de Negocios y Relaciones Internacionales, Coordinador de la Escuela de Marketing y Dirección de Empresas. Universidad Cesar Vallejo S.A.C, Lima - Perú, Email: wzavaletac@ucv.edu.pe, Orcid: https://orcid.org/0000-0003-13539673.

**** Magíster en Administración de negocios - MBA, Universidad César Vallejo - Perú; Magíster en Gestión Pública. Especialista en Administración estratégica, Marketing y en Contrataciones para el Estado. docente investigador en posgrado en la Universidad César Vallejo S.A. C, Lima - Perú, Email: gzarate@ucv.edu.pe, Orcid: https://orcid.org/0000-0002-0565-0577 


\title{
Performance of subnational governments in public investment management, Peru
}

\begin{abstract}
The purpose of this research was to analyze the performance of subnational governments in the management of public investment in Peru. The study was of a basic, non-experimental type, with a qualitative approach, it was developed through the documentary review of various sources relevant and relevant to the research object. The results indicate that subnational governments present an inadequate performance in the management of public investments, due to diverse factors such as management capacity, since there are notorious gaps in the execution of investment spending, inadequate personnel and political interests that affect the level of execution in non-electoral years. Regional governments present worse performance of investment management than local government, since the decrease is $5.53 \%$ and $1.30 \%$ respectively.
\end{abstract}

Keywords: Subnational governments; public investment; public investment management; state performance

\section{Introducción}

El bienestar de los ciudadanos es la máxima finalidad en el ámbito de la gestión pública, la misma que, en miras de un entorno estatal moderno, reúne esfuerzos para el logro de productos y resultados a fin de aminorar brechas diversas identificadas por los sectores nacionales que coadyuven a mejorar la calidad de vida de sus habitantes.

Por tal razón, toma realce la estrategia de descentralización que vienen desarrollando los países, convirtiéndose en un mecanismo que busca multiplicar la presencia estatal en la ciudadanía con la finalidad de conocer problemáticas específicas y adaptar la provisión de servicios gubernamentales a las mismas; con la descentralización, el Estado busca priorizar intervenciones sectoriales que se ajusten a carencias específicas de cada territorio, incentiva competitividad entre los gobiernos descentralizados, generando la autogestión para el manejo y provisión eficiente de los servicios; por otro lado, posibilita la innovación en la medida que hay más de un nivel de gobierno involucrado en el diseño de soluciones a las carencias observadas (Horst Von, 2018).

La situación en la que se gestionan las inversiones públicas es crítica, pues, acorde a los estudios de Organisation for Economic Co-operation and Development (OECD, 2019); las herramientas gubernamentales son vitales $\mathrm{y}$, en los últimos 10 años, la inversión pública ha decrecido, presentando una tasa de $-0.5 \%$ por parte del gobierno central y $-1.1 \%$ en los gobiernos subnacionales; siendo de importancia resaltar, que una proporción del recurso de inversiones superior al $40 \%$ es administrada por los gobiernos 
subnacionales (OECD, 2019).

Lo citado resulta preocupante, pues evidencia que los gobiernos subnacionales no cuentan con el desempeño adecuado para gestionar sus inversiones, dado que gran parte de los recursos asignados no llegan a ser ejecutados para tangibilizar la infraestructura y los servicios que requieren los ciudadanos; situación que resulta contradictoria, puesto que los gobiernos subnacionales tienen mayor proximidad a los habitantes y por ende conocen mejor sus carencias (Torres y Alarcón, 2018). Trabajar en un territorio específico debería de significar mayores índices de efectividad en su desempeño en general.

Si los gobiernos nacionales y subnacionales adoptaran mejores prácticas para la ejecución de infraestructura a través de inversión pública, podrían lograr ahorro de aproximadamente el $40 \%$ del costo, ello representa un aproximado de 1 billón de dólares para la próxima década (Hadden, 2017). Las mejoras en dichas prácticas permitirán hacer efectiva la ejecución de dichos recursos en beneficios de los habitantes, quienes disfrutaran de infraestructura como medio de generación de servicios públicos.

Sí, analizar el desempeño de los gobiernos subnacionales en la gestión de inversión pública, es el objetivo de la investigación. Para ello es necesario generar reflexiones académicas sobre el tema abordado y explorar de manera sistemática fuentes actuales y relevantes para ampliar el conocimiento en dicho tema (Fernández, 2020), buscando desde el plano sustantivo o básico $\mathrm{p}$ ahondar en nuevos conocimientos (Ñaupas et al. 2018).

Se proyectó un abordaje legítimo y verdadero a nivel de contenidos, respetando el método científico. La investigación asumió el enfoque cualitativo para realizar interpretaciones de una realidad mediante la indagación (Hernández y Mendoza, 2018) y se optó por la revisión documental, apoyada en la revisión de documentos físicos y electrónicos de contenido pertinente y variado para describir el tema abordado (Arias, 2020), tomando como punto de partida la problemática identificada (OECD, 2019; Hadden, 2017; Torres y Alarcon, 2018).

\section{Gobiernos Subnacionales}

En el Perú, el gobierno central busca hacer frente a los desequilibrios de la nación mediante la distribución vertical de recursos. Ejerce controles pertinentes a fin de cimentar el aseguramiento del desempeño subnacional eficiente para afrontar a las carencias de sus habitantes. Cabe precisar, que dicho abono de recursos, se trabaja como un proceso continuo, en el que prima la evaluación en base a la previsibilidad y ejecución efectiva del gasto, sustentada en instancias de transparencia multinivel (Ahmad y Searle, 2005). En este contexto, el nivel central supervisa niveles subnacionales, buscando afrontar con efectividad sus problemáticas territoriales específicas.

$$
\text { Estos niveles intermedios }
$$

y primarios del estado, se ven fortalecidos debido a los procesos de descentralización gubernamental que amplían el espectro de provisión e intervenciones variadas. Sin embargo, estos niveles pese a contar con autonomía, aún presentan demasiada dependencia del gobierno central, sobre todo, en materia de transferencia de recursos. Esto dificulta correcciones en las disparidades en su desempeño; 
puesto que no cuentan con capacidad necesaria, en primera instancia, para recaudar recursos, y luego para ejecutar gastos responsables por falta de capacidad administrativa y técnica, además por temor de autoridades regionales y locales de asumir riesgos - responsabilidades económicas y políticas en la recaudación de ingresos (Fretes y Ter-Minassian, 2015).

En países de América Latina y el Caribe, uno de los principales retos recae en elevar la profesionalización del servicio civil, especialmente en niveles subnacionales. En muchos de estos países, el empleo público carece orientación al mérito, aunado a esto la poca calificación de los trabajadores que hacen parte de estas instancias, incluyendo algunos protegidos por acuerdos laborales y contractuales, como es el caso de los nombramientos - contratos por afinidades políticas, lo que en el mediano y largo plazo afecta gravemente la gobernanza y sus resultados en dimensiones sociales y económicas (OECD, 2020).

Este escenario demuestra, el bajo nivel de consecución de los principios de gobernanza pública: participación, transparencia, cuentas rendidas, integridad y ética y conduce a deficiencias generalizadas en el aparato gubernamental, y reduce la confianza de las personas en el Estado. La percepción de corrupción e integridad pública, son factores determinantes en la confianza ciudadana para con el gobierno, pues existen brechas notorias en la lucha contra la pobreza, desigualdad, servicios e infraestructura de calidad, ocasionando descontento generalizado (Murtin et al. 2018). Resulta vital fortalecer las entidades y trabajar en una adecuada implementación de políticas que actúe sobre intereses y necesidades ciudadanas.

En este contexto, se puede afirmar que en los gobiernos de América Latina y el Caribe, la productividad de instancias públicas es afectada tanto por los bajos niveles de competencia e innovación, como por evidencias de fuerte vínculo entre baja calidad del recurso humano y corrupción (OECD, 2016). Esta combinación de factores afecta notoriamente la ejecución de actividades y proyectos, lo que se ve traducido en deficientes niveles de ejecución del gasto público y por ende, en una baja atención de las necesidades de la ciudadanía.

A nivel de ejecución de las actividades por parte de los gobiernos subnacionales, es evidente que no se cumple la totalidad de las mismas, y según plantea el Centro Latinoamericano para el Desarrollo Rural (2015), en América Latina, se evidencia un grave déficit en sus capacidades de análisis y pensamiento estratégico, falta de priorización de agendas urgentes y visión de plazo medio, lo que conlleva a diagnosticar y brindar orden de prelación adecuado en su gestión para elaborar planes de programas, inversiones, contrata en general y cuerpos normativos para el desarrollo del territorio.

En cuanto a la coordinación de esfuerzos entre el gobierno nacional y subnacionales, en Perú esta se muestra limitada o débil. No se logra un marco regulatorio coherente que permita promover buenas prácticas en asuntos regulatorios y de administración; por ello se muestra imperativa la necesidad de fomentar apropiadamente el uso de cuerpos normativos basados en el diagnóstico, evaluación y valoración subnacional en todas sus capas departamentales, provinciales y distritales (OCDE, 2018). 
Lo anterior, obstaculiza la articulación interinstitucional, en parte porque los gobiernos subnacionales carecen de profesionales que entienden apropiadamente la multicausalidad de los procesos que se deberían de ejecutar en el campo de sus competencias por lo que es necesario fomentar estilos de gestión de co-responsabilidad entre divisiones organizativas a fin de mitigar cuellos de botella y brindar atención inmediata ante algún obstáculo que impida el cumplimiento ideal de metas y el efectivo proceso de ejecución presupuestal.
Como se observa en el gráfico 1 , cuya data fue hallada en la consulta amigable del portal de transparencia económica del Ministerio de Economía y Finanzas, en Perú, los gobiernos locales vienen logrando una ejecución presupuestal inferior a los gobiernos regionales, estos últimos presentan una tasa promedio de crecimiento en la ejecución en $0.23 \%$, mientras que los gobiernos locales presentan una tasa de decrecimiento promedio igual al $-0.18 \%$ en los últimos 5 años para el logro de los productos de sus diversas categorías presupuestales y sectorizadas.

\section{Gráfico 1 \\ Ejecución del gasto en actividades por parte de gobiernos subnacionales}

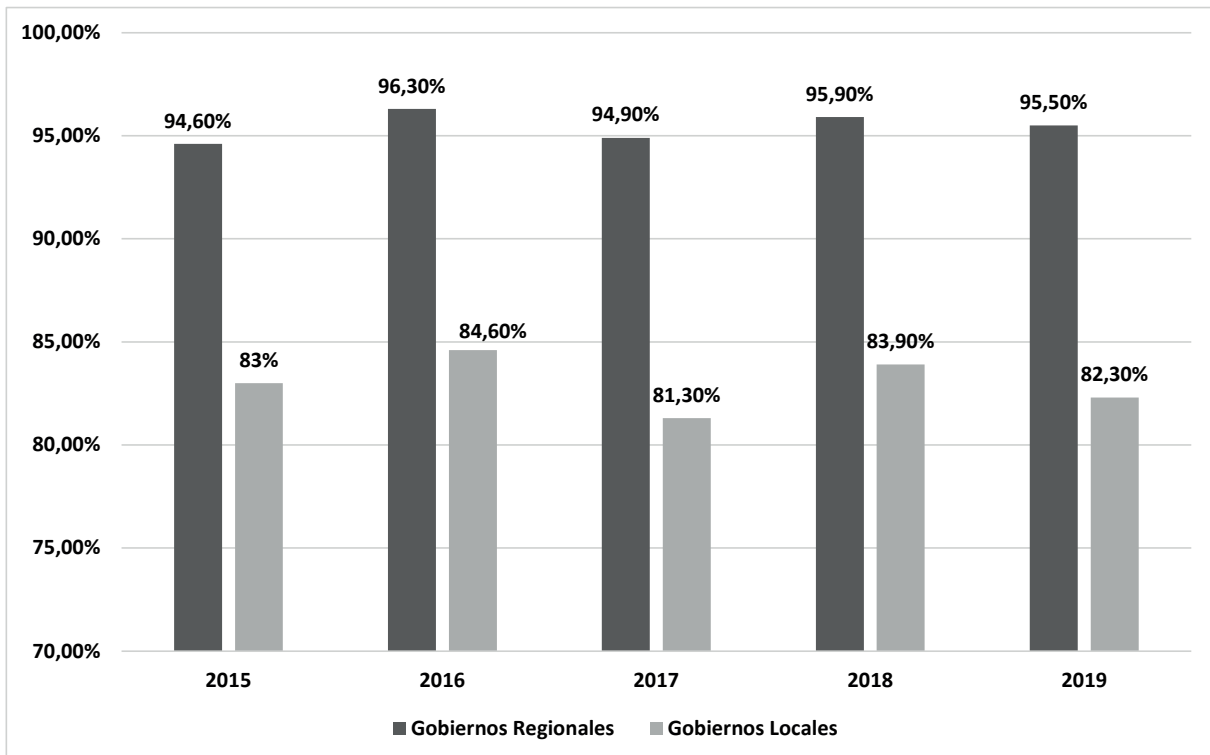

Fuente: Ministerio de Economía y Finanzas (2020). 
Escobar Carmelo, Keny Rodrigo; Terry Ponte, Otto Franklin; Zavaleta Chávez, Walter Enrique; Zárate Ruiz, Gustavo Ernesto

Desempeño de los gobiernos subnacionales en la gestión de inversión pública del Perú

En el gráfico 2, se muestran la cantidad de recursos no ejecutados en los años respectivos a su ejercicio. Hallados en la consulta amigable, de la resta del Presupuesto institucional modificado y compromiso anual como fase de ejecución del gasto público en actividades.

\section{Gráfico 2 \\ Brechas de ejecución del gasto en actividades por parte de gobiernos subnacionales}

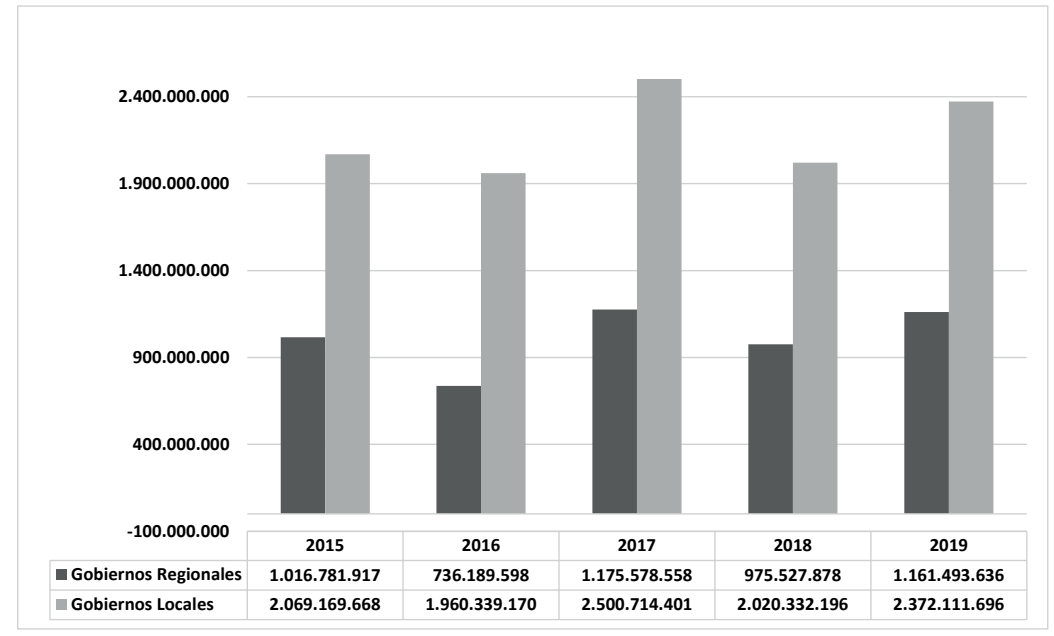

Fuente: Ministerio de Economía y Finanzas (2020)

Las brechas de ejecución del gasto son más pronunciadas en los gobiernos locales, producto de la diferencia entre los recursos asignados totalizados en el presupuesto institucional modificado y el registro del Sistema Integrado de Administración Financiera (SIAF) en la fase de compromiso anual, siendo este un reflejo de acuerdo entre entidades y proveedores previa certificación del presupuesto para su posterior obligación de pago.

En el año 2019, se identificó la mayor brecha de 2'372'111,696 de soles por parte de gobiernos locales; la estimación de los últimos 5 años demuestra que los gobiernos regionales han presentado brecha de 5'065'571,587 de soles, mientras que en los gobiernos locales la situación es más crítica pues esta brecha supera el doble de lo hallado en gobierno regionales con una brecha de 10'922'667,131 de soles; en materia de ejecución financiera, el desempeño de los gobiernos subnacionales no es el apropiado; la situación más agravante es de los gobiernos locales.

Actualmente, en Perú, existen 26 gobiernos regionales y 1846 gobiernos locales, de los cuales 196 son municipalidades provinciales; sin embargo, estas trabajan con muy poca autonomía presupuestaria y financiera, además que, en términos generales, 
los gobiernos subnacionales presentan debilidad institucional para el manejo administrativo y técnico, lo que complica lograr eficiencia en sus propósitos gubernamentales, incluso en materia de contrataciones.

Al 2015, el $20 \%$ de contratos programados no llegaron a ser adjudicados oportunamente; a pesar de existir comités de inversiones en los gobiernos regionales, la priorización de inversiones no es del todo adecuada pues no se respeta a cabalidad la programación multianual de la inversión pública, situación que se agrava en gobiernos locales dado que la prelación de intervenciones se da, en muchos casos, con intenciones políticas y no técnicas. Ello explica por qué en muchos municipios más del $35 \%$ de los recursos de inversión pública se gestionan como bolsa de recursos o genéricos, reasignados a lo largo del año fiscal por falta de selección con criterios vinculantes a mitigación de brechas de calidad y cantidad de servicios (Oliva, 2018).

Acorde a lo citado, es necesario mencionar que esta falta de priorización traducida en un mal manejo de las finanzas públicas por parte de los gobiernos subnacionales propicia la alteración de la calidad del gasto puesto que al no evidenciarse capacidad de planeación, y para evitar el retorno de recursos al gobierno nacional, ejecutan gasto de forma inadecuada en los últimos meses del ejercicio.

En lo que respecta al gasto subnacional, como porcentaje del gasto del gobierno general en América Latina, al año 2017 fue de 47,9\%, en Brasil; de $44 \%$, en Argentina; del $36,5 \%$, en Colombia; del $36,5 \%$, en Perú; del 33,9\%, en México y del 32,9\%, en Bolivia; otros datos relevantes se muestran en las remuneraciones pues los gobiernos locales solo gastan el $10,5 \%$ del presupuesto en ello; mientras que los gobiernos regionales, el 47,7\%; mientras que la comparación del gasto en bienes y servicios es del $15 \%$ a nivel regional y a nivel nacional asciende a $27 \%$; el ingreso de nuevas autoridades subnacionales sin experiencia en gestión pública para el año 2019 pone en mayor evidencia la falta de capacidad que existen en las instancias gubernamentales intermedias (CEPAL, 2019)

No necesariamente mayor ejecución en el gasto refleje más productos e impactos adecuados, por el contrario, las ineficiencias pueden significarle al Perú más del $2 \%$ del producto bruto interno (PBI) significando 15 millones de soles aproximadamente (Instituto Peruano de Economía, 2018). Los gobiernos subnacionales suelen ejecutar sus recursos en los últimos meses del año, sin embargo, existen épocas donde estas cifras cambian radicalmente dado que los gobiernos locales, en años donde hay elecciones, elevan sus gastos equivalentes al $4,2 \%$ del PBI y, en los años restantes, llegan al $3,7 \%$ del $\mathrm{PBI}$, generado, principalmente, por el aumento de alrededor del $50 \%$ de gastos en inversión pública $(E$ Comercio, 2018).

\section{Gestión de la inversión pública}

La gestión de inversiones públicas responde a un enfoque de gobernanza orientado a la adecuada administración de gastos para la ejecución de infraestructura estatal estratégica y eficiente; se compone de logros y lecciones aprendidas desde 1950 en materia de inversión pública (Japan International Cooperation Agency, 2018). 
Escobar Carmelo, Keny Rodrigo; Terry Ponte, Otto Franklin; Zavaleta Chávez, Walter Enrique; Zárate Ruiz, Gustavo Ernesto

Desempeño de los gobiernos subnacionales en la gestión de inversión pública del Perú

Dentro del modelo de gestión gubernamental, elementos como personas son de suma relevancia en la gestión de inversión pública; permiten operar gobiernos subnacionales. Sin embargo, a la fecha, sigue siendo un punto sensible puesto que no entrenan a los equipos profesionales y técnicos de primera línea. La mayoría no cumple con las habilidades y capacidades requeridas para ocupar el puesto que conducen; acompañada de una gestión estratégica y planificación regional débil que afecta la gobernanza institucional.

De acuerdo con el Centro Latinoamericano para el Desarrollo Rural (2015), las metas no son diseñadas adecuadamente y mucho menos se logran cumplir con ellas en su totalidad, reflejo de ello también son las decisiones tomadas en torno a la gestión de proyectos de inversión y la gobernanza que se ejerce en el ciclo de inversiones bajo su competencia, evidenciando la necesidad de mayor articulación inter e intranivel gubernamental para el logro de desarrollo económico sostenible en las jurisdicciones de los gobiernos subnacionales.

Otro factor que influye en la gestión de las inversiones públicas en Perú es el político (gráfico 3) en relación al ciclo político que culminó en el 2014, la data fue presentada en porcentajes por el diario peruano El Comercio.

\section{Gráfico 3}

\section{Ejecución de la inversión pública de los gobiernos locales acorde a temporadas electorales}

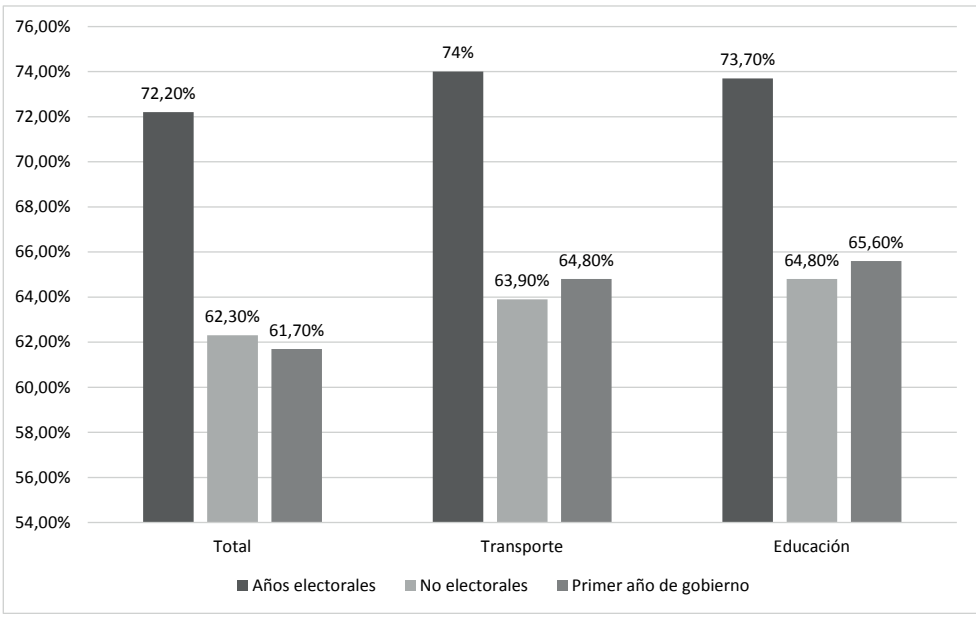

Fuente: El Comercio (2018) 
Es evidente según los datos reflejados en el gráfico 3, que en función al total de presupuesto en inversiones públicas, este se ejecuta al $61.70 \%$ en el primer año de gobierno, siendo los sectores con mayor ejecución los de transporte y educación con $64.80 \%$ y $65.60 \%$, respectivamente (El Comercio, 2018).

El nivel de ejecución incrementa considerablemente en estadios de año electoral, siendo un incremento en $10.50 \%$ en total; incrementa $9.20 \%$ en proyectos de transporte e incrementa en $8.10 \%$ en inversiones del sector educativo. Es cuando se cuestiona el real desempeño o capacidad de los gobiernos locales para administrar proyectos pues si pueden reunir más esfuerzos en años electorales, podrían tranquilamente desplegar estrategias y gestiones similares en los años anteriores a dichos eventos ya que el fin de toda unidad gubernamental es bien común mediante la atención de carencias prioritarias en la ciudadanía y no la de satisfacer intereses políticos; si esta se consigue como fruto de una adecuado accionar del aparato estatal en competencia, la perspectiva cambia, ya que el enfoque potenciador de la gestión de inversiones crea un mejor escenario para los equipos políticos para que puedan ser reelegidos para gobernar puesto que la inversión pública, al tangibilizarse en obras, se hace visible y apreciable por la ciudadanía, los mismos que suelen ponderar la evaluación de sus gobiernos por la calidad de sus obras (Cavalcante, 2015).

Según Núñez et al. (2020), las secuelas de gobernabilidad se dan a raíz de la población pues existe aceptación moderada a pesar de las graves consecuencias en materia de minería, ejecución de proyectos de inversión, y situaciones variadas en sectores como salud, transporte entre otros. Tal es así, que Morillo et al. (2020) refiere que es vital empoderar la participación ciudadana, respecto a temas de planeación y ejecución de obras o inversiones públicas, para así asegurar aciertos en el desarrollo de dichas intervenciones, para ello es necesario que los ciudadanos, a través del derecho a voto, elijan representantes adecuados no solo para ejecutar inversiones, también para el cumplimiento de políticas públicas prioritarias.

La inversión pública cumple un rol sumamente relevante pues es el principal motor interno de crecimiento en las naciones; existe suficiente evidencia de ello en países de diferentes características macroeconómicas, tal es así que las inversiones efectuadas generan mayores impactos frente a otras tipologías de gasto estatal (Izquierdo et al. 2018).

Dicho escenario revela esperanza para reducir las brechas identificadas por sectores y jurisdicciones; no obstante, el rol institucional ha de ser repotenciado pues las entidades rigen la planificación, diseño e implementación de sus mecanismos de inversión pública; por otro lado, los sistemas de inversión pública y sus unidades rectoras han de optimizar sus medios y procesos para que esta forma de gasto pública se traduzca en impacto social y económico.

No obstante, la gestión y avance de los Sistema Nacional de Inversiones Públicas (SNIP) no es homogéneo en América Latinayel Caribe(ALC), puesaún existen falencias de gestión y eficiencia; es necesario resaltar que las inversiones gubernamentales en infraestructura se deben priorizar y tangibilizar de forma adecuada pues estas permiten cimentar el desarrollo sostenido e inclusivo en las 
Escobar Carmelo, Keny Rodrigo; Terry Ponte, Otto Franklin; Zavaleta Chávez, Walter Enrique; Zárate Ruiz, Gustavo Ernesto

Desempeño de los gobiernos subnacionales en la gestión de inversión pública del Perú

comunidades, además de ser uno de los sectores más sensibles a los SNIP; sin embargo, la calidad de infraestructura en ALC se encuentra por debajo de lo esperado según el nivel de stock de capital pues en los últimos 15 años la inversión incrementó; pero aún sigue por debajo del desempeño logrado por los demás países emergentes (Ardanaz, Briceño y García, 2019).

Al respecto, la gestión de inversión pública adolece en la región dadas las evaluaciones de la eficiencia de los sistemas de inversión pública; se encontraron resultados alarmantemente bajos en recursos humanos idóneos, criterios de selección de proyectos, cumplimiento temporal, evaluación expost y seguimiento, sistemas de soporte. Además, en el Perú, resalta la necesidad de fortalecer las guías estratégicas vinculadas a la articulación con las directrices y políticas de los niveles de gobierno superiores, respetando criterios de rentabilidad social, sostenibilidad, pertinencia, otro factor carente es el rol de legislación, auditorías y manejo de activos, relacionado a las acciones previas para la ejecución de obras sea por contrata o por administración directa referida al desempeño de gestión de abastecimiento y control de requerimientos diversos para la implementación de inversiones (Armendáriz et al. 2016).

Esta problemática en las inversiones se puede apreciar en el avance de ejecución; en el grafico 4, donde se detalla la ejecución en los últimos 5 años según la consulta del portal de transparencia económica en el apartado de sólo proyectos.

\section{Gráfico 4 \\ Ejecución del gasto en inversiones públicas por parte de gobiernos subnacionales}

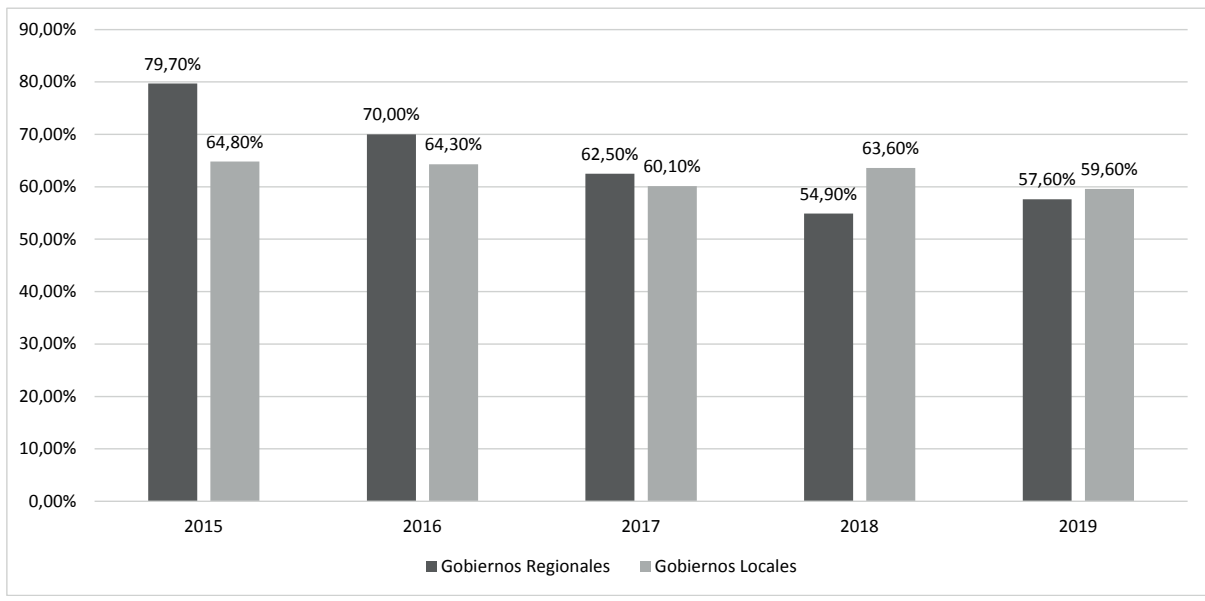

Fuente: Ministerio de Economía y Finanzas (2020).

604 - Esta obra está bajo una licencia de Creative Commons Atribución-Compartirlgual 4.0 Internacional (CC BY-SA 4.0) https://creativecommons.org/licenses/by-sa/4.0/deed.es https://www.produccioncientificaluz.org/index.php/rvg 
En el Perú, los gobiernos locales presentan una ejecución de inversiones por debajo de los gobiernos regionales; sin embargo, en los años 2018 y 2019 , se percibió menor ejecución que los gobiernos locales, tal es así que la tasa promedio de decrecimiento en el nivel de ejecución de los recursos asignados a inversiones es de $-5.53 \%$ en el periodo evaluado para los gobiernos regionales; mientras que en los gobiernos locales dicha tasa es de $-1.30 \%$, por lo que se pone en evidencia el descuido que existe por parte de los gobiernos subnacionales en la gestión de los proyectos de inversión pública y el control de las finanzas de los mismos, siendo los gobiernos regionales quienes presentan peor desempeño que los gobiernos locales en cuanto a la proporción de recursos asignados se refiere.

En el gráfico 5, se muestran con detalle las brechas de ejecución del ejercicio de los últimos 5 años, cuyos resultantes son la diferencia del presupuesto institucional modificado y el compromiso anual como fase de la ejecución del gasto en inversiones públicas.

\section{Gráfico 5 \\ Brechas de ejecución del gasto en inversiones por parte de gobiernos subnacionales}

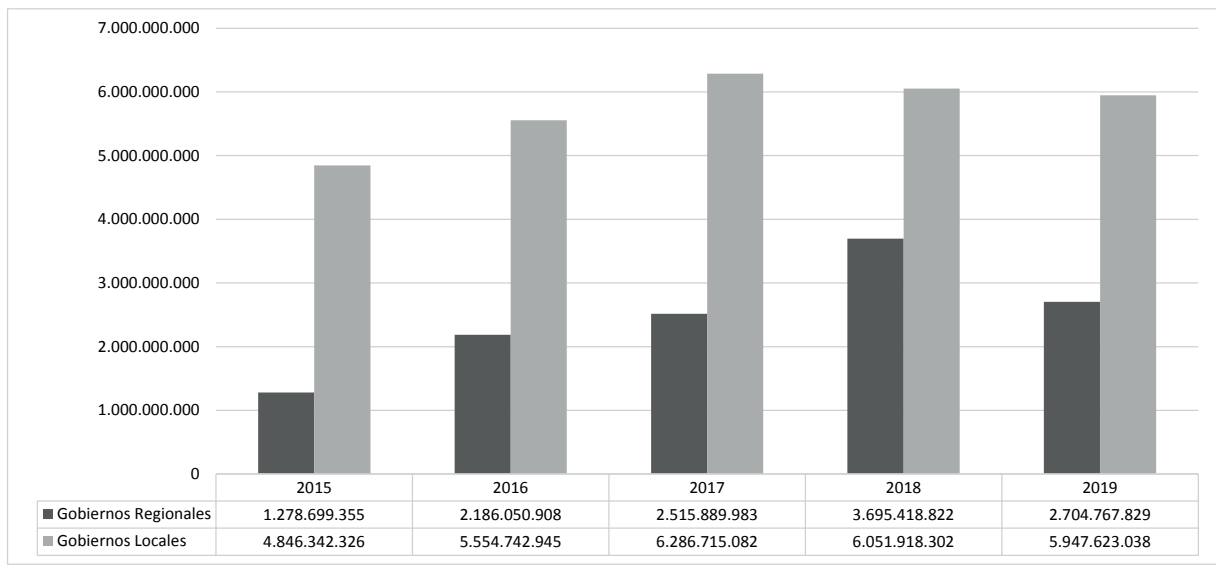

Fuente: Ministerio de Economía y Finanzas (2020).

Las brechas de ejecución de inversiones públicas son más elevadas en los gobiernos locales, dicho cálculo es resultado de la diferencia entre los recursos asignados totalizados en el presupuesto institucional modificado en solo proyectos y el registro del Sistema Integrado de Administración Financiera (SIAF) en la fase de compromiso anual, siendo este un reflejo de acuerdo entre 
entidades y proveedores o contrata de ejecución de obras, previa certificación de crédito presupuestario para su posterior administración de pago.

Enelaño2017, seidentificóla mayor brecha de 6'286'715,082 de soles por parte de gobiernos locales, la estimación de los últimos 5 años demuestra que los gobiernos regionales han presentado brecha totalizada de 12'380'826,897 de soles; mientras que, en los gobiernos locales, la situación es más agravante ya que esta brecha supera el doble de lo hallado en gobiernos regionales con una brecha de 28'687'341,693 de soles; en materia de ejecución financiera de inversiones, el desempeño de los gobiernos subnacionales dista mucho de la eficacia y eficiencia del gasto público, siendo la situación más agravante por parte de los gobiernos locales, tal es así que frente a la ejecución de recursos por actividades, el desempeño de ejecución de inversiones presenta brecha en soles de $144.41 \%$ más por parte de los gobiernos regionales y $162.64 \%$ más por parte de los gobiernos locales.

Es necesario mencionar las principales falencias que existen en el ciclo de gestión de inversiones en América Latina y el Caribe, en la etapa de planeación de inversiones, son débiles los esfuerzos, pues es dispar el nivel nacional con sectorial, estos últimos son más dispersos, inadecuada armonización entre inversión nacional y subnacional, baja capacidad en el proceso de diseño y evaluación de proyectos.

En lo que respecta a selección, los principales problemas son proyectos incorporados al presupuesto sin previa evaluación, financiación por medios extrapresupuestarios. Por otro lado, en la etapa de implementación, resalta la inapropiada contratación y abuso de recurrentes contratos directos, incumplimiento temporal, bajo nivel de ejecución, sobredimensionamiento. En la etapa de evaluación expost, no existen análisis de costo beneficio ante y post ejecución, en líneas generales, las auditorias son mínimas (Ardanaz et al. 2019).

En función de lo anterior, las estrategias de inversión pública requieren ser fortalecidas en instancias subnacionales para hacer más efectiva la coordinación; los criterios de elección y priorización deben ser de acceso público; en lo que respecta a la contrata, resulta necesario que las compras y obras sean más abiertas y transparentes para mitigar posibles actos de corrupción; en lo que respecta a gestión, es necesario fortalecer los medios de seguimiento en carteras de proyectos priorizadas con criterios técnicos suficientes, así como estratificar la evaluación y auditorías; para ello, es necesario mejorar la interoperabilidad de los sistemas administrativos y funcionales intervinientes a la inversión pública, además de hacer más accesible la información a todo nivel, así como trabajar en el fomento de la transparencia de las inversiones para la consulta ciudadana.

\section{Conclusiones}

Fruto de la revisión y análisis de las fuentes, se arribaron a los siguientes hallazgos: en líneas generales, los gobiernos subnacionales presentan dependencia elevada del gobierno central, reflejada en la baja capacidad para obtener ingresos, lo que dificulta la gestión de recursos para atender carencias de la ciudadanía en sus territorios específicos.

En relación a ello, es necesario 
resaltar la ejecución de recursos por parte de los gobiernos subnacionales que carecen de capacidad técnica y administrativa, fruto de la falta de profesionalización del servicio civil, además operan inadecuadamente el aparato estatal de su competencia pues el planeamiento y gestión de productos no presenta priorización de agendas urgentes. Tal es así que los gobiernos locales presentan un nivel de ejecución inferior en lo que actividades se refiere frente a gobiernos regionales pues su decrecimiento en los últimos 5 años es de $-0.18 \%$, siendo la cantidad de recursos no ejecutados oportunamente superior a diez mil millones de soles.

En lo que respecta a la gestión inversión pública, los gobiernos subnacionales poseen equipos débiles pues sus profesionales y técnicos carecen de habilidades y conocimientos necesarios para operar el sistema de inversión pública, esto acompañado de una gestión estratégica inapropiada. Otro factor importante que incide en la gestión de inversión pública es el entorno político, en especial los gobiernos locales dado que estos se caracterizan por no ejecutar adecuadamente sus recursos de inversión; sin embargo, en años electorales, incrementan su ejecución en aproximadamente $10 \%$ por lo que resulta cuestionable el despliegue de capacidades en años no electorales.

Los gobiernos regionales presentan peor desempeño de la gestión de inversiones que los gobiernos locales ya que el decrecimiento es $5.53 \%$ y $1.30 \%$, respectivamente; la consulta demostró también que son débiles los esfuerzos de planificación y selección de proyectos, excediendo el uso de medios extrapresupuestarios; otros factores que obstaculizan la adecuada gestión de inversiones son la inadecuada contratación, contratos dirigidos, incumplimiento temporal y de calidad de infraestructura, las estrategias de seguimiento no surten efecto puesto que no diseñan carteras priorizadas de inversiones, lo que afecta otras variables como el presupuesto participativo y calidad de vida de los ciudadanos; en síntesis, los sistemas de inversión pública están mal operados por profesionales no ajustados al perfil deseado, intereses políticos y una gestión débil por parte de los gobiernos subnacionales.

\section{Referencias bibliograficas}

Ahmad, E. y Searle, B. (2005). On the Implementation of transfers to Subnational Goverments. International Monetary Fund. https:// papers.ssrn.com/sol3/papers. cfm?abstract id=887999

Ardanaz, M., Briceño, B. y García, L. (2019). Fortaleciendo la gestión de las inversiones en América Latina y el Caribe: Lecciones aprendidas del apoyo del BID a los Sistemas Nacionales de Inversión Pública (SNIP). Banco Interamericano de Desarrollo. Estados Unidos. http:// dx.doi.org/10.18235/0001952

Arias, J. (2020). Métodos de investigación on line. Biblioteca Nacional del Perú. Primera edición. Perú. https:// www.tecnologias-informacion.com/ investigacion-mercado-online.html

Armendáriz, E., Contreras, E., Orozco, S. y Parra, G. (2016). La eficiencia del gasto de inversión pública en América Latina. XXI Congreso Internacional sobre la Reforma del Estado y de la Administración Pública. Chile. https:// bit.ly/3hlX609

Cavalcante, P. (2015). Vale a pena ser um bomprefeito? Comportamento eleitoral e reeleição no Brasil. Opinião 
Escobar Carmelo, Keny Rodrigo; Terry Ponte, Otto Franklin; Zavaleta Chávez, Walter

Enrique; Zárate Ruiz, Gustavo Ernesto

Desempeño de los gobiernos subnacionales en la gestión de inversión pública del Perú

Pública. 21(1), 87-104. Recuperado de: $\quad$ https://www.scielo.br/pdf/op/ v21n1/0104-6276-op-21-01-00087. $\underline{\mathrm{pdf}}$

Centro Latinoamericano para el Desarrollo Rural. (2015). Fortalecimiento de capacidades institucionales de gobiernos intermedios para la gestión $y$ gobernanza territorial. Experiencias con gobiernos subnacionales en la región andina. Banco Interamericano de Desarrollo. Bolivia. Recuperado de: https://bit.ly/3qVgmMl

El Comercio. (02 de Julio del 2018). Gobiernos locales ejecutan gastos a última hora para verse mejor. https:// elcomercio.pe/economia/peru/ gobiernos-locales-gastando-ultimahora-verse-mejor-noticia-532240noticia/?ref $=$ ecr

Fernández, V. (2020). Tipos de justificación en la investigación científica. Espíritu Emprendedor TES. 4(3), 65-76. https://doi.org/10.33970/ eetes.v4.n3.2020.207

Fretes, V. y Ter-Minassian, T. (2015). Descentralizando los ingresos fiscales en América Latina, Por qué y cómo. Banco Interamericano de Desarrollo. https://publications. iadb.org/es/publicacion/16934/ descentralizando-los-ingresosfiscales-en-america-latina-por-que-ycomo

Hadden, D. (2017). 7 Reasons why public investment management is critical. Canadá: Free Balance. https://www.freebalance.com/ public-financialmanagement/7reasons-why-public-investmentmanagement-is-critical/

Hernández, R. y Mendoza, C. (2018). Metodología de la Investigación. Las rutas cuantitativa, cualitativa y mixta. McGraw Hill
Horst Von, B. (2018). Antecedentes para una reforma en Chile en materia de financiamiento regional. Libertad y Desarrollo. Serie Informe Económico. https://lyd. org/wp-content/uploads/2018/12/ sie-273-descentralizacion-fiscalantecedentes-para-una-reforma-enchile-en-materia-de-financiamientoregional-noviembre2018.pdf

Instituto Peruano de Economía. (2018). La importancia de decidir. https://www.ipe.org.pe/portal/laimportancia-de-decidir/

Japan International Cooperation Agency. (2018). Public Investment Management Handbook for Capacity Development. Industrial Development and Public Policy Department Governance Knowledge Management Network. Japón. https:// www.jica.go.jp/activities/issues/ governance/ku57pq00001wwbnaatt/strengthen public investment management capacity handbook e.pdf

Ministerio de Economía y Finanzas. (2017). El nuevo sistema de inversión pública. https://www.mef.gob.pe/ contenidos/inv publica/docs/invierte/ INVIERTE.PE.pdf

Ministerio de Economía y Finanzas. (2020). Consulta amigable. Consulta de Ejecución del Gasto. https:// apps5.mineco.gob.pe/transparencial Navegador/default.aspx

Morillo Flores, J., Huachallanqui Salcedo, J., Palacios Garay, J., \& Uribe Hernández, Y. C. (2020). Gobernabilidad y participación ciudadana en el desarrollo local de Lima, Perú. Revista Venezolana De Gerencia, 25(92), 1313-1329. https:// doi.org/10.37960/rvg.v25i92.34264

Murtin, F., Fleischer, L., y Siegerink, V. (2018). Trust and its determinants: Evidence from the Trustlab 
experiment. OECD. Statistics Working Papers. Washington. https:// doi.org/10.1787/869ef2ec-en

Núñez Lira, L. A., Valentín Loayza, J. E., Alfaro Mendives, K. L., y Bonilla Dulanto, E. K. (2020). Gobernanza, representación política y desafección democrática en el Perú. Revista Venezolana de Gerencia. 25(92), 1331346. https://produccioncientificaluz. org/index.php/rvg/article/view/34265

Ñaupas, H., Valdivia, M., Palacios, J. y Romero, H. (2018). Metodología de la investigación cuantitativa cualitativa y redacción de la tesis. Ediciones de la U. Quinta edición. México. https://corladancash. com/wp-content/uploads/2020/01/ Metodologia-de-la-inv-cuanti-y-cualiHumberto-Naupas-Paitan.pdf

Oliva, C. (2018). Gestión de las Finanzas Públicas en el Perú. Informe de Sistematización de las evaluaciones bajo metodología PEFA realizadas a 10 gobiernos subnacionales. Programa de Cooperación al Desarrollo Económico. Basel Institute on Governance. Perú. https:// baselgovernance.org/publications/ gestion-de-las-finanzas-publicas-enel-peru

Organisation for Economic Co-operation and Development- OECD (2019). Effective public investment acroos levels of government. Implementing the OECD principles. Centre for entrepreneurship, SMES, Regions and cities, Washington. https://www. oecd.org/effective-public-investmenttoolkit/OECD Public Investment Implementation Brochure 2019.pdf

Organisation for Economic Co-operation and Development- OECD (2016). Investing in Integrity for Productivity. Working Party of Senior Public Integrity Officials, Washington. https://www.oecd.org/gov/ethics/ investing-in-integrity-for-productivity. pdf

Organisation for Economic Co-operation and Development- OECD (2020). Panorama de las Administraciones Públicas: América Latina y el Caribe 2020. OECD Publishing, París. http:// dx.doi.org/10.18235/0002232

Organización para la Cooperación y el Desarrollo Económico- OECD (2018). Política Regulatoria en el Perú: Uniendo el Marco para la Calidad Regulatoria, Revisiones de la OCDE sobre reforma regulatoria. https:// dx.doi.org/10.1787/9789264279001es

Torres, K. y Alarcón, M. (2018). Polis, ciudadanos felices y gobernabilidad. Mejores gobiernos, ciudadanos felices. Fondo editorial Universidad César Vallejo.

Izquierdo, A., C. Pessino y G. Vuletin (eds.). (2018). Mejor gasto para mejores vidas: Cómo América Latina y el Caribe puede hacer más con menos. Washington, D.C.: BID. 